\title{
Interactive comment on "Observationally constrained analysis of sea salt aerosol in the marine atmosphere" by Huisheng Bian et al.
}

\section{Anonymous Referee \#1}

Received and published: 6 March 2019

Review of Observationally constrained analysis of sea salt aerosol in the marine atmosphere by Bian et al.

The manuscript presents valuable inter-comparison between modelled sea spray mass concentration/AOD and extensive in situ measurements. The latter is the most valuable component of this manuscript as vertical distributions of sea spray are indeed not commonly available on the large geographical scale. These measurements provide very good basis for the validation of the model, however, they were not used to their full potential in this manuscript as the appropriate sea spray source function was not provided. The main conclusion that AOD cannot be reproduced by the current model, due to wrong sea spray source function (SSSF) size distribution, is somehow disappointing without providing the appropriate one. 
In addition to the point raised above, the appropriate comparison of the three SSSF mentioned here is not presented either; There is no discussion or results in the main text, just some numbers in the supplementary, from which it seems that Emi3 results in a higher bias than other schemes. So it is not exactly clear why it was deemed the best here? Manuscript would really benefit from more elaborate discussion on the scheme comparison as well as on how model results compare to AOD measurements using Emi1 and Emi2 schemes? Results should have short description in the main text and only then reference to supplementary (say at lines 117-119);

Introduction section is pretty much biased on USA references, e.g. Quinn and Bates, 2013 is neither the primary nor the main study showing OM in the sea spray; also all other references are mainly from USA scientists, while there are many sea spray papers from European community that were not even mentioned here; For example, extensive SSSF overview paper by (de Leeuw et al., 2011) is missed. Lines 274-275: requires more information and discussion. Is this 0.03 bias comparable with the overestimation here? If not, what percentage is due to bias and what is due to other reasons; Lines 302-304: I understand that the reference is to mass size distribution here, but radiative effects and cloud formation depend more on the number distribution, not mass. Be clear which distribution you refer to and be specific with the effects; Or Lines 313314 , cloud formation is related to size and number not mass; Conclusion on sea water salinity is not convincing globally (lines 400-403), what is salinity variation in global oceans? It might be important locally or regionally close to less saline seas, but not globally; Similarly with the Polar Regions (lines 403-407), indicate how sea ice is relevant to this global study? Is there a higher discrepancy over Polar Regions, if so state that and show the importance? Elaborate on the conclusion sentence in supplementary 'Furthermore, the three emission algorithms discussed in supplementary section show that the uncertainty among the model simulations is generally less than the difference between model and measurement'. First, algorithms do not show anything,

Interactive comment
Printer-friendly version

Discussion paper 
comparison, maybe, second, does this sentence mean that the discrepancy between model and measurements is larger than the model result variation between different SSSF? Clarify. Authors claim that 'Model sensitivity experiments indicated that the simulated sea salt is better correlated with measurements when the sea salt emission is calculated based on the friction velocity and with consideration of sea surface temperature dependence than that parameterized with the 10-m winds' but these results are not properly discussed or presented in the text. Supplementary figures and tables also do not clearly prove that Emi3 is better than other schemes. Correlation might have improved, but the bias got worse. Can you base the conclusion on correlation only?

\section{Specific comments:}

Line 161: provide the correlation coefficient; Line 57-58: Dall et al., 2017 reference is not in the list; Quinn et al., 2017 paper says that sea spray is not important for cloud formation, so the reference is not appropriate here Fig. 2: add ' 3 ' to superscript in both axis names; Do three significant number have meaning in the correlation coefficient and slopes (are they really so precise?); Fig.2 and lines 188-189: R square is usually presented for model-measurement comparisons, have either R2 or both Lines 245-246: $90 \%$ in the mass, not number, provide reference; Lines 281-283: sentence needs rewriting, simulation occurred in July or measurements over this period were compared? Conclusion cannot be obtained in Fig.5. Fig 5 indicates...? Lines 300301: specify what do you mean by 'small particles are more optically efficient' do they scatter better or worse? It is commonly accepted that large particles scatter better; Also, refer to size ranges when talking about small or large particles (here and everywhere in the manuscript) ; E.G line 312: what is small here; Line 318: efficiency? Line 370: with which SSSF the agreement between model and measurements is remarkable? Line 406: Dall et al. 2017 reference is not in the reference list; Table 1: Emi1, Emi2,... are not described in the text or table caption; Supplementary Line 40: Emi3 is improved for total mass not size distribution Supplementary Lines 70-71: what do you

Printer-friendly version

Discussion paper
Interactive

comment 
mean by shifts? Supplementary Line 75: higher than what? Supplementary Line 82: improvement from 0.5 to 0.54 might be perceived as marginal, no? Why there is such big difference in Atom1 and Atom2 agreements, correlations?

de Leeuw, G., Andreas, E. L., Anguelova, M. D., Fairall, C. W., Lewis, E. R., O'Dowd, C., Schulz, M., and Schwartz, S. E.: Production flux of sea spray aerosol, Rev Geophys, 49, RG2001, doi:10.1029/2010rg000349, 2011.

Interactive comment on Atmos. Chem. Phys. Discuss., https://doi.org/10.5194/acp-2019-18, 2019. 